This is not the version of record. The published version (Gurney, Tom, Brouner, James and Spendiff, Owen (2022) "Twenty-one days of spirulina supplementation lowers heart rate during submaximal cycling and augments power output during repeated sprints in trained cyclists." Applied Physiology, Nutrition, and Metabolism, 47(1), pp. 18-26) is Copyright (C) 2021 Canadian Science Publishing and can be found at https://doi.org/10.1139/apnm-2021-0344 
Title: Twenty-one days of spirulina supplementation lowers heart rate during submaximal cycling and augments power output during repeated sprints in trained cyclists

Author list: Tom Gurney ${ }^{1}$, James Brouner ${ }^{1}$, Owen Spendiff ${ }^{1}$

Author affiliations: (1) School of Life Sciences, Kingston University, Kingston upon Thames, KT1 2EE.

$\begin{array}{ll}\text { Corresponding author contact information: } & \text { Tom Gurney } \\ & \text { Kingston University } \\ & \text { Kingston upon Thames } \\ & \text { KT12EE } \\ & \text { E-mail: T.Gurney@kingston.ac.uk }\end{array}$

Email: T.Gurney@kingston.ac.uk

James.Brouner@kingston.ac.uk

O.Spendiff@kingston.ac.uk 


\title{
Twenty-one days of spirulina supplementation lowers heart rate during submaximal cycling and augments power output during repeated sprints in trained cyclists
}

\begin{abstract}
Spirulina supplementation is reported to improve time to exhaustion and $\dot{\mathrm{V}} \mathrm{O}_{2 m a x}$. However, there is limited information on its influence over the multiple intensities cyclists experience during training and competition. Fifteen trained males (Age $40 \pm 8$ years, $\dot{\mathrm{V}}_{2 \max } 51.14 \pm 6.43$ $\mathrm{ml} / \mathrm{min} / \mathrm{kg}$ ) ingested $6 \mathrm{~g} /$ day of spirulina or placebo for twenty-one days in a double-blinded randomized cross over design, with a fourteen-day washout period between trials. Participants completed a 1-hour submaximal endurance test at 55\% external power output max and a $16.1 \mathrm{~km}$ time trial (day 1), followed by a lactate threshold test and repeated sprint performance tests (RSPTs) (day 2). Heart rate (bpm), Respiratory Exchange Ratio, oxygen consumption $(\mathrm{ml} / \mathrm{min} / \mathrm{kg})$, lactate and glucose ( $\mathrm{mmol} / \mathrm{L})$, time (secs), power output (Watts), and hemoglobin $(\mathrm{g} / \mathrm{L})$ were compared across conditions. Following spirulina supplementation, lactate and heart rate were significantly lower $(\mathrm{P}<0.05)$ during submaximal endurance tests $(2.05 \pm 0.80 \mathrm{mmol} / \mathrm{L}$ Vs $2.39 \pm 0.89 \mathrm{mmol} / \mathrm{L} \& 139 \pm 11 \mathrm{bpm} \mathrm{Vs} 144 \pm 12 \mathrm{bpm})$, hemoglobin was significantly higher $(152.6 \pm 9.0 \mathrm{~g} / \mathrm{L})$ than placebo $(143.2 \pm 8.5 \mathrm{~g} / \mathrm{L})$, and peak and average power were significantly higher during RSPTs $(968 \pm 177$ Watts Vs $929 \pm 149$ Watts \& $770 \pm 117$ Watts Vs $738 \pm 86$ Watts). No differences existed between conditions for all oxygen consumption values, $16.1 \mathrm{~km}$ time trial measures and lactate threshold tests $(\mathrm{P}>0.05)$. Spirulina supplementation reduces homeostatic disturbances during submaximal exercise and augments power output during RSPTs.
\end{abstract}

\section{Novelty bullets:}

- Spirulina supplementation lowers heart rate and blood lactate during $\approx 1$-hour submaximal cycling.

- Spirulina supplementation elicits significant augmentations in hemoglobin and power outputs during RSPTs.

Key words: algae, lactate, heart rate, hemoglobin, peak power, average power 


\section{INTRODUCTION}

The supplementation of spirulina (SP), a cyanobacteria that is commonly referred to as bluegreen algae, is garnering attention. However, at present, the understanding of the ergogenic capabilities of SP are equivocal. This multicomponent species boasts a vast array of vitamins, minerals and proteins (Kalafati et al., 2010) and the exact mechanisms of action behind any ergogenic effect has been difficult to ascertain. Yet, authors have previously reported SP to improve time to exhaustion whilst running (Kalafati et a., 2010; Lu et al., 2006) and $\dot{\mathrm{V}} \mathrm{O}_{2 \max }$ whilst cycling (Kalpana et al., 2017; Hernández-Lepe et al., 2018). Indeed, these previous investigations have primarily focused on SP's antioxidant mechanistic potential during/after exercise by comparing a variety of oxidative stress markers (Kalafati et al., 2010; Lu et al., 2006; Kalpana et al., 2017), albeit with conflicting findings (Pappas et al., 2021; Franca et al., 2010). However, the oxidative stress biomarkers used in the aforementioned studies are considered outdated and lack robustness (Cobley et al., 2017). Some positive in Vitro research has however supported the notion that SP may increase the activity of intracellular antioxidant enzymes, possibly via the activation of the NRF2 signaling pathway (Finamore et al., 2017; Gao et al., 2016). Nevertheless, these findings should be interpreted with caution, particularly as the direct link between antioxidant effects and improvements in exercise tolerance in the previous studies are not clear. Therefore, possible new mechanistic avenues are worthy of consideration when investigating the efficacy of SP supplementation.

Previous SP supplementation research has also reported improvements in hemoglobin $(\mathrm{Hb})$ (Gurney \& Spendiff, 2020; Kelkar et al., 2008; Milasius et al., 2009; Selmi et al., 2011; Ulivar 
et al., 2000). However, thus far, how these increases in $\mathrm{Hb}$ might influence exercise performance has received little attention and remains unanswered. For example, it is well reported that $\mathrm{Hb}$ is indispensable for oxygen transport and cell respiration(Buratti et al., 2015), and even trivial increases in $\mathrm{Hb}$ have been associated with improving oxygen transport to the working muscles which therefore could improve aerobic oxidative capacity and $\dot{\mathrm{V}} \mathrm{O}_{2 \max }$ (Buratti et al., 2015; Mairbäurl, 2013). As such, recent research has reported SP to reduce heart rate and improve oxygen uptake during arm cycling exercise (Gurney \& Spendiff, 2020).

Further investigations have also attempted to distinguish how SP may influence the blood lactate response following exercise; conflicting results have been reported when looking at peak lactate values following time to exhaustion during cycling and running (Lu et al., 2006; Kalpana et al., 2017; Torres-Durán et al., 2012; Hernández-Lepe et al. 2018), whilst Juszkiewicz et al. (2018) reported no differences in lactate after a 2000m rowing time trial. However, to the best of our knowledge, no investigation has investigated how SP may influence the blood lactate response during lower intensities. The possibility of SP reducing metabolic perturbation in the muscle, and therefore metabolic acidosis, may permit submaximal exercise intensities to be performed with lower homeostatic disturbance.

Many cycling races elicit a variety of intensities that characteristically include long lower intensity rides lasting $\geq 1$-hour, time trials and uphill sprint finishes (Bell et al., 2017) whereby each cyclist will have to produce a continuous high-power output. Certainly, producing high power-outputs has previously been reported to be a determinant of sprint cycling performances (Kordi et al., 2019,2020; Dorel et al., 2005). Consequently, having both efficient aerobic and anerobic energy metabolism systems, including the ability to tactically produce repeated sprints whilst using brief recovery periods to buffer the raised acidosis and shuttle lactate associated 
with fatigue, are considered essential to cycling competition performance and training sessions (Santalla et al., 2012).

To date, limited research has been directed into trained cyclists supplementing with SP (Franca et al., 2010) and to the best of our knowledge, no study has investigated how SP may influence performance over multiple cycling intensities. Furthermore, while the optimal dose/duration strategy is yet to be established, with previous research ranging from $1.5 \mathrm{~g} / \mathrm{day}-7.5 \mathrm{~g} /$ day and 7 - 60 days, in line with previous efficacious results by both Kalafati et al., 2010 and Lu et al., 2006, it was decided to supplement $6 \mathrm{~g}$ /day for 21-days. Therefore, the aim of this study was to investigate whether $6 \mathrm{~g}$ /day supplementation of SP for 21-days can influence key physiological measures during submaximal endurance tests, $16.1 \mathrm{~km}$ time trial (TT), lactate threshold, and repeated sprint performance tests (RSPT). It was hypothesised that the supplementation of SP would lower the homeostatic disturbance during submaximal exercise and improve $16.1 \mathrm{~km}$ TT performances, lactate thresholds and RSPTs.

\section{MATERIALS AND METHODS}

\section{Participants}

Fifteen trained, healthy male, experienced cyclists volunteered and gave their written consent to participate in the study (Mean $\pm \mathrm{SD}$; Age $40 \pm 8$ years, Stature $182.3 \pm 6.2 \mathrm{~cm}$, Mass $77.4 \pm$ $6.8 \mathrm{~kg}$, average weekly self-reported Kilometers/hours cycled $198.3 \pm 68.0 \mathrm{~km} / 8.0 \pm 3.0 \mathrm{hrs}$, $\dot{\mathrm{V}} \mathrm{O}_{2 \max } 51.14 \pm 6.43 \mathrm{ml} / \mathrm{min} / \mathrm{kg}$ ). A priori power analysis in $\mathrm{G}^{*}$ Power ${ }^{\circledR}$ software (version 3.1.9.2; Universität Kiel, Kiel, Germany) (Faul et al., 2007) using an alpha of .05 and a power of .80, and using the improvement in heart rate during submaximal arm cranking with SP from our previous work (Gurney \& Spendiff, 2020), indicated that a sample size of 15 participants were required to detect a medium effect for SP supplementation. 
Participants were required to train at least 4-5 hours per week and have a minimum of 2 years of cycling experience. Any cyclist that currently smoked or had a history of cardiovascular disease was excluded. The Faculty of Science, Engineering and Computing Ethics Committee at Kingston University London approved the study in accordance with the Declaration of Helsinki.

\section{Study Design}

In a double-blind randomized cross-over design, participants were required to visit the laboratory on a total of six separate occasions, at the same time of day, following an overnight fast and were asked to refrain from exercise $48 \mathrm{hrs}$ before each visit. Throughout the intervention, participants were instructed to refrain from taking any other supplements, to maintain their regular training regime and to record a $48 \mathrm{hr}$ food and exercise diary prior to the first session, this was replicated prior to each subsequent visit. Adherence to the diary was $100 \%$.

The first visit comprised baseline anthropometric measurements and included a $\dot{\mathrm{V}}_{2 \max }$ test. Additionally, familiarisation of the exercise testing protocols occurred during visit 1 and 2, separated by at least $24 \mathrm{hrs}$, whereby participants completed the protocols in the exact same order as they would post intervention.

Participants were then randomly allocated to either SP (Indigo Herbs Limited - see Table 1 for nutritional composition) or placebo (microcrystalline cellulose) in a counter-balanced design and were instructed to ingest 6 grams (14 capsules: 5 with breakfast, 5 with lunch, 4 with dinner) each day for 21-days. All capsules were visually identical, sealed within 21 small bags 
and were coded and counter-balanced by an independent lab technician. No participants reported any visual or taste differences nor gastrointestinal issues after each supplement and could not guess which supplement, they were on.

The morning after the 21-day supplementation period, participants reported to the laboratories to begin their two-day testing period (visit $3 \& 4$ ). During visit 3, participants were required to complete a 1-hour submaximal endurance test at $55 \%$ of their maximal external power output $\left(\right.$ Mean \pm SD: $205 \pm 37 \mathrm{~W}$ ) achieved at $\dot{\mathrm{VO}}_{2 \max }($ Mean $\pm \mathrm{SD}: 377 \pm 67 \mathrm{~W})$, followed 5-minutes later by a $16.1 \mathrm{~km} \mathrm{TT}$. The $2^{\text {nd }}$ testing day occurred $24 \mathrm{hrs}$ later (visit $4,+2$-day post intervention) and consisted of a lactate threshold test followed 10-minutes later by RSPT (see below for protocols). All testing was always completed with participants sat in the saddle. Each performance test was assessed for test-retest reliability, expressed as CV\%: $16.1 \mathrm{~km} \mathrm{TT} \mathrm{(time}$ taken $-3.6 \%$, power output $-4.2 \%$ ), RSPT (peak power $-3.1 \%$, average power $-1.9 \%$ ), lactate threshold (heart rate $-4.2 \%$, power output $-1.4 \%$ ), 55\% submaximal endurance test (respiratory exchange ratio (RER) - 3.0\%, oxygen consumption $\left(\dot{\mathrm{VO}}_{2}\right)-2.2 \%$, lactate $-4.2 \%$, heart rate $1.6 \%$ ). Between the $4^{\text {th }}$ and $5^{\text {th }}$ visit to the laboratory, there was a minimum interval of 35 days to allow for a 14-day wash out and the subsequent 21-day supplementation period before completing the exact same 2-day testing period (visit $5 \& 6$ ). This daily dosage and wash-out period is similar to previous work (Kalafati et al., 2010). A schematic illustration of the protocol design can be seen in Figure 1.

\section{Baseline measurements \& $\dot{\mathbf{V}} \mathbf{O}_{2 \max }$}

During the first visit, and at the beginning of each subsequent visit, body mass $(\mathrm{kg})$ and a $10 \mu \mathrm{L}$ capillary blood sample for $\mathrm{Hb}(\mathrm{g} / \mathrm{L})$ analysis (HemoCue AB Hb 2001+, Ängelholm, Sweden) was taken. Thereafter, participants were asked to adjust each bike to their preferred saddle 
height, which was recorded and replicated for each subsequent visit. For the $16.1 \mathrm{~km} \mathrm{TT}$, participants used a TT bike (Boardman Bikes Ltd, London, UK) fixed on a Tacx Turbo Trainer (i-Genius T2020, Fisher Outdoor Leisure PLC UK) which was calibrated following the manufacturer's instructions prior to each trial. All other tests were completed on a Peak Bike (Monark Exercise AB, S-432 82 Varberg, Sweden).

For the $\dot{\mathrm{V}}_{2 \max }$ test, respiratory variables were measured (Vyntus CPX; Vyaire Medical GmbH, Germany) continuously and averaged to 15 -second intervals. Heart rate was recorded (Polar Electro Oy, Kempele, Finland) at every minute until volitional fatigue. The test comprised an initial 3-minute warm up period with no resistance $(0 \mathrm{~W})$ at a cadence between 60-70 Revolutions Per Minute (RPM). Each participant then started at $120 \mathrm{~W}$ and was asked to cycle at exactly $80 \mathrm{RPM}$ throughout the test, with $25 \mathrm{~W}$ increments every minute until volitional fatigue or until the cadence could no longer be maintained. The $\dot{\mathrm{V}} \mathrm{O}_{2 \max }$ was determined by the highest $\dot{\mathrm{VO}}_{2}$ value that was recorded from the 15 -second averages and maximal external power output (W) was rounded down to the nearest incremental stage. The maximum $\dot{\mathrm{VO}}_{2 \max }$ and external power output was recorded to subsequently establish each participant's $55 \%$ relative intensity for the 1-hour submaximal endurance tests.

\section{Exercise testing protocols}

\section{Day 1 - Submaximal endurance test and $16.1 \mathrm{~km}$ TT}

Participants' $55 \%$ relative intensity was manually adjusted onto the Peak Bike before being instructed to cycle at exactly 80RPM for 1-hour. Respiratory variables (RER, $\dot{\mathrm{VO}} 2$, and $\left.\mathrm{CO}_{2}\right)$ were recorded continuously, averaged over 15 seconds and subsequently averaged over 20minute intervals for data analysis. Heart Rate and $20 \mu \mathrm{L}$ capillary blood samples (glucose and lactate) were recorded every 20-minutes (20, 40, 60-minutes) (Biosen C-Line Sport, EKF 
$\mathrm{GmbH}$, Germany). Each participant was then given a 5-minute rest whereby water was consumed ad libitum before starting the $16.1 \mathrm{~km}$ TT. The volume of water consumed was subsequently recorded and standardized for the following visits. The TT was executed at a freely chosen RPM, participants were able to see the distance cycled but were blinded to all other variables. $\dot{\mathrm{V}} \mathrm{O}_{2}(\mathrm{ml} / \mathrm{min} / \mathrm{kg})$ and power output (Watts) were continuously measured and averaged for total mean comparative data analysis. Additionally, capillary blood samples were taken at every $4 \mathrm{~km}$ interval $(4,8,12,16 \mathrm{~km})$. Verbal encouragement was provided throughout.

\section{Day 2 - Lactate Threshold test \& Repeated Sprint Performance Tests}

An incremental test modified from Valenzuela et al. (2018) and Bentley et al. (2007) was employed. The test started at $120 \mathrm{~W}$ with increases of $25 \mathrm{~W}$ every 3 -minutes, with each participant maintaining 80RPM. Capillary blood samples and heart rate were collected at rest and in the final 30-seconds of each 3-minute stage. The test was terminated once the participant could no longer maintain a cadence of 80RPM. Lactate threshold was calculated by using the Dmax method in 'Lactate E' software (Newell et al., 2007). Power output and heart rate at the calculated lactate threshold were compared across each condition.

After a 10-minute rest period, each participant was instructed to complete the RSPT which was designed in accordance to previous research employing similar repeated sprint protocols (Jonvik et al., 2018). The test consisted of a 5-minute warm up at 50W (including 2 x 3 second sprints). A 1-minute rest period was then given before starting 3 x 20 second all out sprints. The RSPT was performed with a flying start against an applied fixed load of $8.5 \%$ body mass which automatically dropped once the participant cycled above 90RPM. Each sprint was interspersed by a 4-minute active recovery period whereby the participant was instructed to freely spin at $50 \mathrm{~W}$ and consume water ad libitum. In the $2^{\text {nd }}$ minute of the 4-minute recovery 
periods, capillary blood samples were taken (lactate and glucose). Participants were blinded to all output variables. Average power (AP), peak power (PP) (Watts), cadence (RPM), as well as fatigue indexes (Watts/sec \& power drop \%), were automatically recorded by the Peak Bike Monark software during each repeated sprint for analysis. Overall means, individual RSPT means and total mean cadence across the entire RSPT were used for analysis.

\section{Statistics}

Data are presented as mean \pm SD. All statistical procedures were carried out using IBM SPSS version 26 for windows. All data sets were analyzed for normality using Shapiro Wilk, while Mauchly's test of Sphericity was employed to establish any potential violations. Any violation of sphericity was corrected by using values from the Greenhouse-Geisser. Statistical significance alpha level was set at $\leq 0.05$. Effect sizes (ES) calculated using Partial ETA squared SPSS output, Observed Power and 95\% Confidence Intervals (95\% CI) were used where appropriate. All variables from the submaximal endurance test, RSPT, heart rate and lactate during the first 4 stages of the lactate threshold tests and lactate from the $16.1 \mathrm{~km}$ TT were analyzed using a two-way within subjects repeated measures ANOVA, whereas $\mathrm{Hb}$ were analyzed using a one-way within subjects repeated measures ANOVA, with both ANOVA's using a Bonferroni correction for multiple comparisons to determine any differences. Heart rate and power output at lactate threshold and $16.1 \mathrm{~km}$ TT variables were compared using a paired sample $T$-Test.

\section{RESULTS}

\section{1-hour submaximal endurance tests:}

\section{Lactate}


During the 1-hour submaximal endurance test, the total average blood lactate response was lower following SP supplementation $(2.05 \pm 0.80 \mathrm{mmol} / \mathrm{L})$ compared to placebo $(2.39 \pm 0.89$ $\mathrm{mmol} / \mathrm{L})(\mathrm{P}=0.012, \mathrm{ES}=0.37$, Observed Power $=0.76)$. Post Hoc analysis revealed lactate to be significantly lower between conditions during the first and last 20-minutes, see Figure 2a $(\mathrm{P}=0.021,95 \% \mathrm{CI}=-.61--.05 \& \mathrm{P}=0.005,95 \% \mathrm{CI}=-.67--.14)$. A significant effect for time was detected $(\mathrm{P}<0.05)$, with values at rest being significantly lower than all other time points $(\mathrm{P}<0.05)$. A condition*time interaction was detected $(\mathrm{P}<0.05)$.

\section{Heart Rate}

During the 1-hour submaximal endurance test, heart rate was lower following SP supplementation $(139 \pm 11 \mathrm{bpm})$ compared to placebo $(144 \pm 12 \mathrm{bpm})(\mathrm{P}=0.001, \mathrm{ES}=0.73$, Observed Power $=0.90)$. Post Hoc tests reported SP to yield a significantly lower heart rate at each 20-minute time point, see Figure $2 b$ (20-minutes: $\mathrm{P}=0.001,95 \% \mathrm{CI}=-6.35--2.54,40$ minutes: $\mathrm{P}=0.001,95 \% \mathrm{CI}=-7.50--3.35,60$-minutes: $\mathrm{P}=0.001,95 \% \mathrm{CI}=-8.85--3.61) . \mathrm{A}$ significant effect for time was detected, with both conditions demonstrating increases at every 20-minute interval $(\mathrm{P}<0.05)$. There was no condition*time interaction $(\mathrm{P}>0.05)$.

There was no effect of condition or time for: $\dot{\mathrm{VO}}_{2}(\mathrm{SP}=33.46 \pm 4.48 \mathrm{ml} / \mathrm{min} / \mathrm{kg}$ Vs Placebo= $34.34 \pm 5.61 \mathrm{ml} / \mathrm{min} / \mathrm{kg}, \mathrm{P}=0.127), \operatorname{RER}(\mathrm{SP}=0.93 \pm 0.05 \mathrm{Vs} \mathrm{Placebo}=0.94 \pm 0.03, \mathrm{P}=0.393)$ and glucose $(\mathrm{SP}=3.88 \pm 0.62 \mathrm{mmol} / \mathrm{L}$ Vs Placebo $=3.90 \pm 0.56 \mathrm{mmol} / \mathrm{L}, \mathrm{P}=0.862)$, and no condition*time interaction during the 1-hour submaximal endurance tests $(\mathrm{P}>0.05)$.

\section{$16.1 \mathrm{~km}$ TT:}

There was no difference in TT performance between placebo and SP $(\mathrm{P}=0.436)$. There was no effect of condition or time for: lactate $(\mathrm{P}=0.747)$, glucose $(\mathrm{P}=0.413)$, heart rate $(\mathrm{P}=0.857)$, 
power output $(\mathrm{P}=0.981)$ and $\dot{\mathrm{VO}}_{2}(\mathrm{P}=0.360)$ during the TT (see Table 2$)$, and no condition*time interaction $(\mathrm{P}>0.05)$.

\section{Hemoglobin:}

Following SP supplementation, a significant effect on $\mathrm{Hb}$ was observed $(\mathrm{P}=0.001, \mathrm{ES}=0.43$, Observed Power $=0.97$ ), with Post Hoc tests revealing there to be a significant increase after SP supplementation $(152.6 \pm 9.0 \mathrm{~g} / \mathrm{L})$ in comparison to baseline $(142.8 \pm 6.9 \mathrm{~g} / \mathrm{L}, \mathrm{P}=0.008$, $95 \% \mathrm{CI}=2.55-17.05)$ and placebo $(143.2 \pm 8.5 \mathrm{~g} / \mathrm{L}, \mathrm{P}=0.007,95 \% \mathrm{CI}=2.49-16.17)$. No differences were detected between baseline and placebo $(\mathrm{P}>0.05)$ (see Figure 3).

\section{Repeated Sprint Performances Test:}

\section{Average Power}

During the RSPT, AP was significantly higher following SP supplementation $(770 \pm 117 \mathrm{~W})$ compared to placebo $(738 \pm 86 \mathrm{~W})(\mathrm{P}=0.029, \mathrm{ES}=0.29$, Observed Power $=0.61)$. Post Hoc tests revealed SP to produce a higher AP output during the $1^{\text {st }}(\mathrm{P}=0.007,95 \% \mathrm{CI}=13.39-68.95)$ and $3^{\text {rd }}(\mathrm{P}=0.018,95 \% \mathrm{CI}=7.83-70.33)$ sprint. A condition*sprint interaction was detected $(\mathrm{P}<0.05)$, see Figure 4a.

\section{Peak Power}

During the RSPT, PP was significantly higher following SP supplementation (968 $\pm 177 \mathrm{~W})$ compared to placebo $(929 \pm 149 \mathrm{~W})(\mathrm{P}=0.037, \mathrm{ES}=0.27$, Observed Power $=0.57)$. Post Hoc tests reported SP to produce higher PP during the $1^{\text {st }}(\mathrm{P}=0.006,95 \% \mathrm{CI}=17.73-89.24)$ and $3^{\text {rd }}(\mathrm{P}=0.017,95 \% \mathrm{CI}=10.93-97.80)$ sprint. There was no condition*sprint interaction $(\mathrm{P}>0.05)$, see Figure $4 \mathrm{~b}$. 
For both PP and AP there was a significant effect detected across time in both conditions $(\mathrm{P}<0.05)$.

\section{Lactate}

During the RSPT, the blood lactate response was significantly higher following SP supplementation $(11.69 \pm 3.30 \mathrm{mmol} / \mathrm{L})$ compared to placebo $(10.62 \pm 2.74 \mathrm{mmol} / \mathrm{L})(\mathrm{P}=0.011$ $\mathrm{ES}=0.38$, Observed Power $=0.78$ ). Post Hoc tests revealed blood lactate to be significantly higher during the $1^{\text {st }}(\mathrm{P}=0.015,95 \% \mathrm{CI}=.29-2.33)$ and $2^{\text {nd }}(\mathrm{P}=0.017,95 \% \mathrm{CI}=.25-2.25)$ sprint. A significant effect for time was detected in both conditions whereby lactate increased continuously at every subsequent sprint $(\mathrm{P}<0.05)$. There was no condition*sprint interaction $(\mathrm{P}>0.05)$.

\section{Cadence}

During the RSPT, cadence was significantly higher following SP supplementation (146 \pm 20RPM) compared to placebo $(138 \pm 17 \mathrm{RPM})(\mathrm{P}=0.003, \mathrm{ES}=0.49$, Observed Power $=0.92)$. Post Hoc tests revealed cadence to be significantly higher during the $1^{\text {st }}(\mathrm{P}=0.042,95 \% \mathrm{CI}=$ $.19-8.82)$ and $2^{\text {nd }}(\mathrm{P}=0.005,95 \% \mathrm{CI}=5.55-26.54)$ sprint. There were both a significant effect for time and a condition*time interaction $(\mathrm{P}<0.05)$.

There was no effect of condition or time for: Watts/Second $(\mathrm{SP}=22 \pm 5$ Vs Placebo $=21 \pm 5$, $\mathrm{P}=0.511)$, Power Drop \% $(\mathrm{SP}=44 \pm 8 \% \mathrm{Vs}$ Placebo $=44 \pm 8 \%, \mathrm{P}=0.887)$ and glucose $(\mathrm{SP}=$ $4.76 \pm 0.73 \mathrm{mmol} / \mathrm{L}$ Vs Placebo $=4.49 \pm 0.77 \mathrm{mmol} / \mathrm{L}, \mathrm{P}=0.066)$, and no condition*time interaction. 


\section{Lactate Threshold:}

Power output $(\mathrm{SP}=252 \pm 53 \mathrm{~W}$ Vs Placebo $=246 \pm 55 \mathrm{~W})$ and heart rate $(\mathrm{SP}=158 \pm 8 \mathrm{bpm} \mathrm{Vs}$ $\mathrm{Placebo}=161 \pm 8 \mathrm{bpm})$ were not significantly different $(\mathrm{P}=0.970 \& \mathrm{P}=0.223$, respectively $)$ at the calculated threshold. Heart rate during the first 4-stages $(120 \mathrm{~W}, 145 \mathrm{~W}, 170 \mathrm{~W}, 195 \mathrm{~W})$ of the lactate threshold test was not significantly different between conditions $(\mathrm{P}=0.066,95 \% \mathrm{CI}=$ -5.92 - .22). Equally, there was no difference between conditions for lactate during the first 4stages $(\mathrm{P}=0.549)$.

\section{DISCUSSION}

To date this appears to be the first study to compare key physiological responses during submaximal endurance tests, TTs, lactate thresholds and RSPTs after the supplementation of SP in trained cyclists. The key novel findings of this study were that $6 \mathrm{~g} /$ day supplementation of SP for 21-days significantly reduced heart rate whilst demonstrating a reduced blood lactate response during submaximal exercise intensities (Figure 2). Spirulina supplementation also elicited significant augmentations in $\mathrm{Hb}$ (Figure 3) and power outputs during RSPTs (Figure 4).

\section{Submaximal Endurance Tests}

Following SP supplementation, a continuous lower heart rate and lactate response (Figure 2) in comparison to placebo transpired, consistent with previous research trends (Kalpana et al., 2017; Hernández-Lepe et al., 2018; Gurney \& Spendiff, 2020; Torres-Durán et al., 2012). While the exact physiological mechanism of action may be difficult to establish due to SP's multicomponent properties, a key mechanistic avenue to consider when interpreting these cardiovascular responses is that $\mathrm{Hb}$ significantly increased, consistent with previous literature owed to SP containing iron (Gurney \& Spendiff, 2020; Kelkar et al., 2008; Milasius et al., 
2009; selmi et al., 2011). In clinical SP supplementation studies, Selmi et al. (2011) reported significant increases in $\mathrm{Hb}$ in elderly citizens following a $3 \mathrm{~g}$ /day for 12 -weeks protocol and Uliyar et al. (2000) reported up to an $11 \%$ increase of $\mathrm{Hb}$ in young women following $5 \mathrm{~g} /$ day for 30-days. Kelkar et al. (2008) previously demonstrated that after just a 4g/day two-week SP supplementation period, $\mathrm{Hb}$ in healthy elite marathon runners prior to a race significantly increased. A further positive haemopoietic system trend was also noted by Milasius et al. (2009) following a two-week supplementation of just $2.25 \mathrm{~g} /$ day in 'high performance sportsmen'. With both authors further supporting the notion of good bodily assimilation of iron from SP, whilst also suggesting that SP can combat against the adverse effects from oxidative stress that may occur in erythrocyte membranes (Kelkar et al. 2008). It has been reported that the absence of phytate and oxalate in SP allows for a high iron absorption rate (GutiérrezSalmeán et al., 2015), as both compounds have previously been reported to inhibit iron absorption due to the binding of insoluble complexes within iron (Walter, 1997). Given the importance of optimal iron stores for daily Hb synthesis (Buratti et al., 2015), the possible high iron absorption rate from SP supplementation can play a key role in daily $\mathrm{Hb}$ formation.

However, its plausible to suggest that increases in $\mathrm{Hb}$ following the supplementation of SP in healthy subjects, without an iron deficiency, may have been derived from a different mechanism. Recent work by Engan et al. (2020) demonstrated that 5 mmol nitrate supplementation can stimulate the contraction of the spleen during apneas which can consequently elevate circulatory $\mathrm{Hb}$ by up to $3 \%$. Although previous investigations into SP and nitrate are sparse, previous work by Brito et al., (2018) and Ichimura et al. (2013) may support the notion that SP can increase circulatory nitrate/nitrite whereby it was reported that the phycocyanin constituent found in SP increased nitric oxide availability in the aorta and plasma in rats, and increases the expression of Endothelial Nitric Oxide Synthase (eNOS). 
Considering both aforementioned mechanisms, the elevation of $\mathrm{Hb}$ can cause an ergogenic effect as $\mathrm{Hb}$ plays an indispensable role in transporting oxygen from the lungs to the working muscles and consequently supports the oxidative phosphorylation pathway (Buratti et al., 2015; Mairbäurl, 2013). Hence, augmentations in $\mathrm{Hb}$ have been associated with improving aerobic oxidative capacity during exercise (Mairbäurl, 2013). Indeed, it has been purported that SP may enhance oxidative capacity (Kalpana et al., 2017; Hernández-Lepe et al., 2018; TorresDurán et al., 2012), which may concomitantly attenuate the heart rate and lactate response via an increase in lactate metabolism during the 1-hour submaximal endurance test. Efficient lactate metabolism has recently been highlighted to play an important role in mitochondrial respiration and provides fuel as an energy source (Brooks, 2020). Conversely, the incessant higher blood lactate values in the placebo condition (Figure 2a) indicates an increasing rate of metabolic acidosis during the 1-hour submaximal endurance tests (Robergs et al., 2004). Subsequently, this caused heart rate to increase in order to redress and expel the metabolic unbalance which ultimately resulted in a significant difference to occur over time.

Spirulina contains several constituents which may further support the apparent lower cardiovascular demand seen in the current study when exercising submaximally. These overlapping mechanisms may be influencing similar pathways. Firstly, SP contains phycocyanin, a pigment-protein complex that has previously been reported to increase the expression of Endothelial Nitric Oxide Synthase (eNOS) in rats (Ichimura et al., 2013). Secondly, SP contains arginine (Lafarga et al., 2020), the amino acid precursor to nitric oxide, which has previously been reported to increase muscle blood volume via its endothelium vasodilatory effects (Álveres et al., 2012). Whilst Brito et al. (2018) has demonstrated that the supplementation of SP improves the contractile reactivity of the aorta via increases in nitrite 
production and increases in the antioxidant activity in rats. Though the measurement of these potential mechanisms was omitted, the plausibility of SP possessing vasodilatory mechanistic properties is supported by previous research in humans (Torres-Durán et al., 2012; Miczke et al., 2016).

Despite the apparent amelioration in heart rate and blood lactate response, a similar trend was not observed in the present study when analysing the submaximal $\dot{\mathrm{VO}}_{2}$ results. As such, these findings contradict our previous research whereby SP reduced oxygen demand during submaximal arm cycling (Gurney \& Spendiff, 2020). Importantly, differing muscle groups/exercise modalities, population, duration and intensities make comparisons problematic. Although further research is needed, collectively, these results indicate SP supplementation has several mechanisms which may allow submaximal exercise to be performed with a lower homeostatic disturbance.

\section{Repeated Sprint Performance Tests}

Another novel finding from the study following SP supplementation is that both PP and AP (Figure 4) during the RPSTs significantly increased. The results from the present study are consistent with previous literature whereby SP has been reported to improve peak and average force of the dominant quadricep muscle (Sandhu et al., 2010) and short high intensity exercise (Chaouachi et al., 2020; Kalafati et a., 2010; Lu et al., 2006; Kalpana et al., 2017; HernándezLepe et al., 2008). Indeed, having the ability to produce large power outputs, particularly PP, is an essential prerequisite in competitive cyclists and has previously been reported to be a determinant of performance (Kordi et al., 2019, 2020). Although there was a significantly $(\mathrm{P}=0.011)$ higher blood lactate response during the RSPTs after SP supplementation, this is 
consistent with previous work by Lu et al., (2006), and might indeed reflect an increase in glycolytic flux and/or increased clearance via improved blood flow.

The authors currently have little understanding on the potential mechanistic reasonings behind an improvement in PP, particularly as previous research has highlighted that both neuromuscular and morphological changes are related to increases in PP. However, our results demonstrated that cadence (RPM) at PP was significantly higher after SP supplementation perhaps suggestive that the changes observed could be neuromuscular rather than morphological (Dorel et al., 2005). Further, previous in Vitro research suggests that the phycocyanin content in SP can increase in the scavenging and activity of intracellular antioxidant enzymes, possibly via the activation of the NRF2 signaling pathway (Finamore et al., 2017; Gao et al., 2016). Undeniably, the measurement of both these changes were omitted as they were beyond the scope of the study, nonetheless the results can help elucidate further suggested physiological mechanisms of action following SP supplementation.

\section{Time Trial and Lactate Thresholds}

The non-significant findings from the TT in the present study are perhaps consistent with previous literature whereby SP seems to improve exercise performance during short high intensity exercise tests such as $\dot{\mathrm{V}}_{2 \max }$ whilst cycling (Kalpana et al., 2017; Hernández-Lepe et al., 2018), time to exhaustion whilst running (Kalafati et al., 2010; Lu et al., 2006), and during short intermittent exercise modalities such as RSPTs (as seen in the current study). The $16.1 \mathrm{~km}$ TT in both conditions lasted on average 26-minutes and according to our heart rate and power output data, was performed above the participants lactate threshold for the duration (see results 'lactate threshold' and Table 2). Therefore, it is plausible to suggest that SP does not influence exercise performance that is performed for a substantial time over the threshold. For 
example, the 1-hour endurance test was performed on average at $205 \pm 37 \mathrm{~W}$ and $139 \pm 11 \mathrm{bpm}$, nearly $50 \mathrm{~W}$ and $20 \mathrm{bpm}$ lower than the calculated threshold in the SP condition $(252 \pm 53 \mathrm{~W}$ and $158 \pm 8 \mathrm{bpm})$, whereby it was evident that SP supplementation alleviated the physiological demand in comparison to placebo (Figure 2).

Spirulina did not generate a significant improvement in heart rate and lactate at the calculated lactate threshold. There were also no significant differences in heart rate $(\mathrm{P}=0.066,95 \% \mathrm{CI}=$ $5.92-.22)$ or lactate $(\mathrm{P}=0.549)$ during the first 4 lower intensity stages of the lactate threshold test. These results conflict with previous research whereby it has been reported that SP can reduce heart rate at the onset blood lactate accumulation during an incremental test (HernándezLepe et al., 2018). However, these findings were from overweight populations, making comparisons between the two studies difficult. Overall, SP's influence on lactate metabolism capabilities is conflicting, particularly at high intensities (Lu et al., 2006; Juszkiewicz et al., 2018). Whilst there seems to be some trends in this study during lower intensities and previous research (Lu et al., 2006; Hernández-Lepe et al., 2018; Torres-Durán et al., 2012), further research is warranted to establish the optimal intensity SP is most efficacious at.

\section{Limitations}

Interpretation of the results should come with caution due to several key limitations associated with the study. Firstly, a major limitation is that the SP powder used in this study was not independently batch tested for its nutritional compositional analysis. Secondly, although every attempt was made to recruit 'healthy' participants, monitoring of the nutritional status of participants throughout the intervention was not completed. It is not yet established whether SP may be most beneficial to athletes who may be initially nutritionally deficient due to the algae containing multiple proteins, vitamins and minerals. Indeed, this may explain any 
potential 'responders' and 'non responders' in future studies. Another key limitation to the study is that we were unable to successfully recruit any female participants; female athletes are severely underrepresented in research and therefore the results from the current study may lack application to the wider cycling community. Future research should ensure to include both sexes. Future research should also attempt to focus on specific constituents found in SP and their potential conclusive mechanisms of action. Further comparisons should also be made in order to find the optimal dosage, exercise modality and intensity. Finally, it's important to acknowledge that since the $16.1 \mathrm{~km}$ TT, lactate threshold and RSPT were conducted after fatiguing exercise, the findings might not necessarily be translatable to the same test initiated from an unfatigued state.

\section{AUTHOR STATEMENTS}

\section{Acknowledgements}

The authors would like to thank the participants for their time and effort in completing the study.

\section{Conflict of Interest statement}

The authors declare that there is no conflict of interest.

\section{Contributors' statement}

The study was designed by T. Gurney, J. Brouner and O. Spendiff; data were collected by T. Gurney; data were analysed by T. Gurney; data interpretation and manuscript preparation were 
undertaken by T. Gurney, J. Brouner and O. Spendiff. All authors approved the final version of the paper.

\section{Funding statement}

The authors declare no specific funding for this work.

\section{Data availability statement}

The datasets generated during and/or analysed during the current study are available from the corresponding author on reasonable request. 


\section{References}

Bell P, Furber M, Van Someren K, Antón-Solanas A, Swart J. (2017) The Physiological Profile of a Multiple Tour de France Winning Cyclist. Medicine \& Science in Sports \& Exercise, 49(1):115-123. doi: 10.1249/MSS.0000000000001068.

Bentley DJ, Newell J, Bishop D. (2007) Incremental Exercise Test Design and Analysis. Sports Medicine, 37(7):575-86 doi: 10.2165/00007256-200737070-00002

Brito, A., Silva, A., de Oliveira, C., de Souza, A., Ferreira, P., de Souza, I., da Cunha Araujo, L, et al. (2020) Spirulina platensis prevents oxidative stress and inflammation promoted by strength training in rats: dose-response relation study. Scientific Reports, 10(1) https://doi.org/10.1038/s41598-020-63272-5

Brooks GA. (2020) Lactate as a fulcrum of metabolism. Redox Biology, 101-454. PMID: 32113910

Buratti P, Gammella E, Rybinska I, Cairo G, Recalcati S. (2015) Recent Advances in Iron Metabolism. Medicine \& Science in Sports \& Exercise, 47(8):1596-1604. doi: 10.1249/MSS.0000000000000593.

Chaouachi, M., Gautier, S., Carnot, Y., Bideau, N., Guillemot, P., Moison, Y., Collin, T., Vincent, S, et al. (2020) Spirulina platensis Provides a Small Advantage in Vertical Jump and Sprint Performance But Does Not Improve Elite Rugby Players' Body Composition. Journal of Dietary Supplements, 1-16. https://doi.org/10.1080/19390211.2020.1832639

Cobley, J. N., Close, G. L., Bailey, D. M., \& Davison, G. W. (2017). Exercise redox biochemistry: Conceptual, methodological and technical recommendations. Redox biology, 12, 540-548. doi: 10.1016/j.redox.2017.03.022

Dorel, S., Hautier, C., Rambaud, O., Rouffet, D., Van Praagh, E., Lacour, J., Bourdin, M., (2005) Torque and Power-Velocity Relationships in Cycling: Relevance to Track Sprint Performance in World-Class Cyclists. International Journal of Sports Medicine, 26:739-746 https://doi.org/10.1055/s-2004-830493

Engan H, Patrician A, Lodin-Sundström A, Johansson H, Melin M, Schagatay E (2020) Spleen contraction and $\mathrm{Hb}$ elevation after dietary nitrate intake. $J$ Appl Physiol 129(6):1324-1329 doi: 10.1152/japplphysiol.00236.2020.

Faul, F, Erdfelder, E, Lang, A, Buchner, A. (2007) G*Power 3: A flexible statistical power analysis program for the social, behavioral, and biomedical sciences. Behavior Research Methods, (2), 175-191. doi: 10.3758/bf03193146.

Finamore, A., Palmery, M., Bensehaila, S., Peluso, I. (2017). Antioxidant, immunomodulating, and microbial-modulating activities of the sustainable and ecofriendly spirulina. Oxidative medicine and cellular longevity. doi: $10.1155 / 2017 / 3247528$.

Franca, G., Silva, A., Costa, M., Moura Junior, J., Nóbrega, T., Gonçalves, M., Asciutti, 1. (2010) Spirulina does not decrease muscle damage nor oxidative stress in cycling 
athletes with adequate nutritional status. Biology of Sport, 27(4):249-253 https://doi.org/10.5604/20831862.927489

Gao, Y., Liu, C., Wan, G., Wang, X., Cheng, X., Ou, Y. (2016). Phycocyanin prevents methylglyoxal-induced mitochondrial-dependent apoptosis in INS-1 cells by Nrf2. Food \& function, 7(2), 1129-1137. doi: 10.1039/c5fo01548k.

Gurney, T \& Spendiff, O. (2020) Spirulina supplementation improves oxygen uptake in arm cycling exercise. European Journal of Applied Physiology, https://doi.org/10.1007/s00421-020-04487-2

Gutiérrez-Salmeán G, Fabila-Castillo L, Chamorro-Cevallos G. (2015) Nutritional And Toxicological Aspects Of Spirulina (Arthrospira). Nutricion Hospitalaria, 32(1):3440. doi: 10.3305/nh.2015.32.1.9001.

Hernández-Lepe M, López-Díaz J, Juárez-Oropeza M, Hernández-Torres R, WallMedrano A., Ramos-Jiménez A. (2018) Effect of Arthrospira (Spirulina) maxima Supplementation and a Systematic Physical Exercise Program on the Body Composition and Cardiorespiratory Fitness of Overweight or Obese Subjects: A Double-Blind, Randomized, and Crossover Controlled Trial. Marine Drugs, 16(10):364. doi: 10.3390/md16100364.

Ichimura, M., Kato, S., Tsuneyama, K., Matsutake, S., Kamogawa, M., Hirao, E., Miyata, A., Mori, S, et al. (2021). Phycocyanin prevents hypertension and low serum adiponectin level in a rat model of metabolic syndrome. Nutrition Research, 33(5), 397 - 405 https://doi.org/10.1016/j.nutres.2013.03.006

Jonvik, K., Nyakayiru, J., Van Dijk, J., Maase, K., Ballak, S., Senden, J., Van Loon, L., Verdijk, L. (2018) Repeated-sprint performance and plasma responses following beetroot juice supplementation do not differ between recreational, competitive and elite sprint athletes. European Journal of Sport Science, 18(4), 524-533. https://doi.org/10.1080/17461391.2018.1433722

Juszkiewicz, A., Basta, P., Petriczko, E., Machaliński, B., Trzeciak, J., Łuczkowska, K., Skarpańska-Stejnborn, A. (2018) An attempt to induce an immunomodulatory effect in rowers with spirulina extract. Journal of the International Society of Sports Nutrition, 15(1). https://doi.org/10.1186/s12970-018-0213-3

Kalafati, M., Jamurtas, A., Nikolaidis, M., Paschalis, V., Theodorou, A., Sakellariou, G., Koutedakis, Y., Kouretas, D. (2010) Ergogenic and Antioxidant Effects of Spirulina Supplementation in Humans. Medicine \& Science in Sports \& Exercise, 42(1):142151. https://doi.org/10.1249/mss.0b013e3181ac7a45

Kalpana K, Kusuma D, Lal P, Khanna G. (2017) Impact of Spirulina on Exercise Induced Oxidative Stress and Post Exercise Recovery Heart Rate of Athletes in Comparison to a Commercial Antioxidant. Food \& Nutrition Journal, 4(4). doi: 10.29011/25757091. 100039

Kelkar G, Subhadra K, Chengappa R. (2008) Effect of Antioxidant Supplementation on Hematological Parameters, Oxidative Stress and Performance of Indian Athletes. Journal of Human Ecology, 24(3):209-213. doi: 10.1080/09709274.2008.11906116 
Kordi, M., Folland, J., Goodall, S., Menzies, C., Patel, T., Evans, M., Thomas, K., Howatson, G. (2020) Cycling-specific isometric resistance training improves peak power output in elite sprint cyclists. Scandinavian Journal of Medicine \& Science in Sports, 30(9), 1594-1604. https://doi.org/10.1111/sms.13742

Kordi, M., Folland, J., Goodall, S., Haralabidis, N., Maden-Wilkinson, T., Sarika Patel, T., Leeder, J., Barratt, P, et al. (2019) Mechanical and morphological determinants of peak power output in elite cyclists. Scandinavian Journal of Medicine \& Science in Sports, 30(2), 227-237. https://doi.org/10.1111/sms.13570

Lafarga T, Fernández-Sevilla J, González-López C, Acién-Fernández F. (2020) Spirulina for the food and functional food industries. Food Research International, 137:109356. doi: 10.1016/j.foodres.2020.109356.

Lu H, Hsieh C, Hsu J, Yang Y, Chou H. (2006) Preventive effects of Spirulina platensis on skeletal muscle damage under exercise-induced oxidative stress. European Journal of Applied Physiology, 98(2):220-226. doi: 10.1007/s00421-006-0263-0.

Mairbäurl H. (2013) Red blood cells in sports: effects of exercise and training on oxygen supply by red blood cells. Frontiers in Physiology, 4 .

https://doi.org/10.3389/fphys.2013.00332

Miczke A, Szulińska M, Hansdorfer-Korzon R, Kręgielska-Narożna M, Suliburska J, Walkowiak J, Bogdański P. (2016) Effects of spirulina consumption on body weight, blood pressure, and endothelial function in overweight hypertensive Caucasians: a double-blind, placebo controlled, randomized trial. European Review for Medical and Pharmacological Sciences, 20(1):150-156, PMID: 26813468.

Milasius K, Malickaite R, Dadeliene R. (2009) Effect of spirulina food supplement on blood morphological parameters, biochemical composition and on the immune function of sportsmen. Biology of Sport, 26(2):157-172.

Newell J, Higgins D, Madden N, Cruickshank J, Einbeck J, McMillan K, McDonald R. (2007) Software for calculating blood lactate endurance markers. Journal of Sports Sciences, 25(12):1403-1409. doi: 10.1080/02640410601128922

Pappas A, Tsiokanos A, Fatouros IG, Poulios A, Kouretas D, Goutzourelas N, Giakas G, Jamurtas AZ. (2021). The Effects of Spirulina Supplementation on Redox Status and Performance Following a Muscle Damaging Protocol. International Journal of Molecular Sciences, 22(7), 3559. doi: 10.3390/ijms22073559.

Robergs, R., Ghiasvand, F. and Parker, D. (2004) Biochemistry of exercise-induced metabolic acidosis. The American Journal of Physiology-Regulatory, Integrative and Comparative Physiology,287(3):R502-R516. doi: 10.1152/ajpregu.00114.2004.

Sandhu J, Dheera B, Shweta S. (2010) Efficacy of spirulina supplementation on isometric strength and isometric endurance of quadriceps in trained and untrained individuals - a comparative study. Ibnosina Journal of Medicine and Biomedical Sciences, 2:79 doi: 10.4103/1947-489X.210974 
Santalla, A, Earnest, C, Marroyo, J., Lucia, A. (2012) The Tour de France: An Updated Physiological Review. International Journal of Sports Physiology and Performance, 7(3):200-209. doi: 10.1123/ijspp.7.3.200.

Selmi C, Leung PS, Fischer L, German B, Yang CY, Kenny TP, Cysewski GR, Gershwin ME. (2011) The effects of Spirulina on anemia and immune function in senior citizens. Cellular \& Molecular Immunology, 8(3):248-254. doi: 10.1038/cmi.2010.76

Torres-Durán, P., Ferreira-Hermosillo, A., Ramos-Jiménez, A., Hernández-Torres, R., Juárez-Oropeza, M. (2012) Effect of Spirulina maxima on Postprandial Lipemia in Young Runners: A Preliminary Report. Journal of Medicinal Food, 15(8):753-757. doi: 10.1089/jmf.2011.0309

Uliyar, M., Alefia, S., Uma, I., Panam, P. (2000). The effect of Spirulina supplementation on blood haemoglobin levels of anaemic adult girls. Journal of Food Science and Technology (Mysore), 37(6), 642-644. https://doi.org/10.1038/cmi.2010.76

Valenzuela P, Morales J, Foster C, Lucia A, de la Villa P. (2018) Is the Functional Threshold Power a Valid Surrogate of the Lactate Threshold? International Journal of Sports Physiology and Performance, 13(10):1293-1298. doi: https://doi.org/10.1123/ijspp.2018-0008

Walter P. (1997) Effects of Vegetarian Diets on Aging and Longevity. Nutrition Reviews, 55(1):S61-S68. doi: 10.1111/j.1753-4887.1997.tb06106.x

Wu Q, Liu L, Miron A, Klímová B, Wan D, Kuča K. (2016) The antioxidant, immunomodulatory, and anti-inflammatory activities of Spirulina: an overview. Archives of Toxicology, 90(8):1817-1840. doi: 10.1007/s00204-016-1744-5 


\section{Figure Legends:}

Figure 1. A schematic illustration of the study design.

Figure 2. Lactate (mmol/L) (A) and heart rate (bpm) (B) values during the 1-hour submaximal endurance test following the 21-day supplementation of spirulina or placebo. ${ }^{*}$ signifies $\mathrm{P}<.05$ between conditions. $\dagger$ \# signifies both a spirulina $(\dagger)$ and placebo $(\#)$ within trial increase $\mathrm{P}<.05$. \$ signifies condition*time interaction effect.

Figure 3. Hemoglobin $(\mathrm{g} / \mathrm{L})$ at baseline and following the consumption of placebo and spirulina. * signifies $\mathrm{P}<.05$ between spirulina and placebo, and spirulina and baseline.

Figure 4. Average power (A) and peak power (B) (Watts) during the repeated sprint performance test following the 21-day supplementation of spirulina or placebo. Black solid line represents group mean, dotted grey line represents individual responses, * signifies $\mathrm{P}<.05$ between conditions. $\dagger \#$ signifies both a spirulina $(\dagger)$ and placebo $(\#)$ within trial increase $\mathrm{P}<.05$. \$ signifies condition* sprint interaction effect.

Table 1. Nutritional composition of spirulina $(100 \mathrm{~g} \& 6 \mathrm{~g})$

Table 2. Mean \pm SD for performance variables measured during the $16.1 \mathrm{~km} \mathrm{TT}$. 